\title{
EL TRABAJO COMO SIGNIFICANTE Y SIGNIFICADO EN LA SOBRECUALIFICACIÓN LABORAL DE LAS MUJERES BRASILEÑAS EN BARCELONA
}

\author{
Work as a signifier and meaning in the over-qualification \\ of Brazilian women in Barcelona
}

Gemma Antón Ramos*

\begin{abstract}
Resumen. Este artículo se centra en los significados del trabajo que se contextualizan en el proceso de migración autónoma de las mujeres brasileñas en Barcelona (España), y en la sobrecualificación como significante de su situación laboral. La particularidad de ambas circunstancias implica tener en cuenta tanto los aspectos estructurales como subjetivos. Así, el análisis se fundamenta en la identificación social y en la identidad en relación al trabajo. Ello contribuye a ampliar la comprensión sobre el concepto de trabajo más allá de la racionalidad económica, y a evidenciar la noción de sobrecualificación como un mecanismo de mantenimiento de la jerarquía del trabajo.
\end{abstract}

Palabras clave: mujeres migrantes, migración autónoma, desigualdades, trabajo, sobrecualificación.

\begin{abstract}
This article is focused on the meanings of work that are contextualized in the process of independent migration of Brazilian women in Barcelona (Spain), and in the overqualification as a signifier of their work situation. The particularity of these circumstances involves taking into account both, structural and subjective aspects. Thus, the analysis is based on social identification and identity regarding work. This contributes to understanding of the concept of work beyond economic rationality, and highlighting the notion of overqualification as a tool for maintaining the hierarchy of work.
\end{abstract}

Keywords: migrant women, independent migration, inequalities, overqualification, work.

* Universitat de Barcelona. Barcelona, España. 


\section{Introducción}

El objetivo de este artículo, en el que se basa mi trabajo de investigación ${ }^{1}$, es analizar las prácticas de trabajo que llevan a cabo las mujeres brasileñas en Barcelona (España) en relación a la situación de sobrecualificación laboral en la que se encuentran. La migración autónoma que realizan está influenciada por ciertas desigualdades estructurales en relación a las normas patriarcales, a las cuestiones raciales ${ }^{2}$ y socioeconómicas, siendo éstos dos últimos aspectos sesgados a su vez por el género. En estos casos, la dinámica migratoria tiene un efecto inverso al de la vinculación familiar en el contexto de origen y representa una ruptura con dicho entorno en el proceso de reproducción social.

En esta línea, resulta interesante analizar las prácticas de las mujeres en el contexto de trabajo en Barcelona desde diversas perspectivas que se interrelacionan, por una parte la del trabajo como fuente de subsistencia y por otra, como construcción identitaria individual y social. Ello implica tener en cuenta la subjetividad como un factor importante principalmente por dos motivos. En primer lugar porque la emigración de las mujeres involucradas en el estudio no se genera a partir de motivaciones únicamente económicas y laborales, mientras que por otro lado el concepto de sobrecualificación laboral, tal como argumentaré en adelante, no puede considerarse desvinculado del aspecto subjetivo.

Tener en cuenta el aspecto subjetivo en estos procesos implica reflexionar sobre qué es lo que el trabajo significa más bien que lo que es en sí como definición del concepto. El enfoque que adopto se determina entre la actividad económica y la actividad social contemplando los aspectos políticos, socioculturales e identitarios. Ello se fundamenta, por una parte, en la identificación de las mujeres como inmigrantes en el contexto de acogida. Las representaciones que se plasman sobre ellas afectan su posicionamiento en el ámbito del trabajo, que es entendido a partir de la significación social en base a ciertas ideologías y prácticas institucionales tal como argumento en el tercer apartado del presente artículo. En esta línea, por lo que respecta a las prácticas de las mujeres en cuestión voy a considerar la situación de sobrecualificación laboral en la que éstas se encuentran. Ello se fundamenta en base a cómo las

\footnotetext{
1 Este artículo se basa en mi estudio de tesis Reproducción social, migración y generaciones. El caso de las mujeres brasileñas en Barcelona. Se enmarca en el programa de doctorado en Antropología Social de la Universitat de Barcelona, así como en el trabajo de investigación realizado con el Grupo de Estudios sobre Reciprocidad -GER de la misma universidad, concretamente dentro del proyecto CONJUST, y con el Grupo de Estudios en Antropología y Significados del Trabajo -GREAST del Instituto Catalán de Antropología -ICA. La investigación está financiada por el MECD mediante una beca de Formación de Profesorado Universitario -FPU.

2 Las desigualdades respecto a la raza son referidos a la discriminación que afecta a las mujeres afro-brasileñas en relación a los aspectos racistas y sexistas (LINO, Nilma. Educação, raça e gênero: relações imersas na alteridade, p. 75).
} 
mujeres se inscriben en las formas discursivas sobre la alteridad y sus simbolismos en el contexto de acogida, en la medida en que los sistemas dominantes actúan selectivamente dificultando la efectiva inserción en el contexto laboral, lo que se materializa en la sobrecualificación. Por otro lado, el apartado cuarto se centra en el aspecto identitario por lo que se refiriere a los significados que se generan a partir de las relaciones sociales en el contexto de trabajo, que son influidos por los valores económicos, sociales e individuales. En esta parte voy a tratar cómo es vivido el trabajo que las mujeres desenvuelven y los significados que interactúan en dichas experiencias.

Tener en cuenta la importancia de las relaciones sociales en el ámbito del trabajo y su construcción categórica conlleva adoptar una perspectiva popular del concepto más allá del economicista, es decir, considerando las percepciones y las prácticas de las personas comunes. Si bien este enfoque puede dificultar la concreción en la definición del concepto de trabajo resulta útil para ampliar su comprensión desde la aproximación antropológica, arrojando luz sobre cómo el trabajo es experienciado a partir de las aproximaciones subjetivas y los condicionamientos socioculturales.

El trabajo es un elemento de valoración social básico para la construcción de la identidad que cobra sentido en un contexto concreto a partir de ciertas determinaciones y relaciones sociales ${ }^{3}$. Más allá del aspecto económico, la noción de trabajo que voy a tratar se manifiesta como fuente de valores culturales y sociales construidos, es decir, como un concepto relacional que está incrustado en las relaciones sociales. En los contextos concretos donde el trabajo se lleva a cabo es donde se expresan las relaciones sociales existentes, así las diferencias de clase, etnia y género son creadas, reproducidas y estructuradas a través de éste ${ }^{4}$. En esta línea, la idea de trabajo que se relaciona en los casos de las mujeres brasileñas en Barcelona es aplicada considerando una categorización social discriminatoria, la de trabajadoras inmigrantes.

Cabe destacar que el trabajo familiar no remunerado ${ }^{5}$, que llevan a cabo mayoritariamente las mujeres, carece de visibilidad y reconocimiento social no siendo contemplado en las lógicas productivas del mercado. En esta línea, la conceptualización del (no)trabajo familiar se fundamenta en base al paradigma

\footnotetext{
WADEL, Cato. The hidden work of everyday life, p. 381; COMAS D'ARGEMIR, Dolores. Trabajo, género, cultura: la construcción de desigualdades entre hombres y mujeres, p. 34.

4 COMAS D'ARGEMIR, op. cit., p. 13; NAROTZKY, Susana. Antropología económica: nuevas tendencias, p. 118-119; SINCLAIR, M. Thea. Women, work and skill: economic theories and feminist perspectives, p. 1; JULIANO, Dolores. Excluidas y marginales, p. 39-40.

5 El concepto de 'trabajo familiar' no remunerado revela que se extiende más allá del trabajo doméstico, contemplando actividades como servicios para el hogar, tareas de gestión, organización, trámites, distribución de tareas, etc., que se articulan entre el estrato social, los servicios y la familia en lo referente a la reproducción humana (BORDERÍAS, Cristina. Strategie della libertà: storie e teorie del lavoro femminile, p. 62-63).
} 
del trabajo asalariado que influye en la idea negativa del trabajo doméstico y de cuidado, considerado 'irracional' y desvinculado de la productividad' . Estas prácticas se relacionan con el valor social del trabajo realizado por mujeres y, a su vez, con el grado de (des)prestigio que representan. En la línea de estas observaciones, voy a conceptualizar las tareas domésticas, de cuidado y familiares como trabajo, basándome en las postulaciones sobre el debate del trabajo doméstico ${ }^{7}$. Ello implica por tanto considerarlo como una situación de sobrecualificación.

La imagen desvalorizada del trabajo considerado 'femenino' da a su vez un grado de valor sobre su especificidad que es aplicado a todo tipo de trabajo que desarrollan las mujeres. En esta línea cabe destacar que en los casos estudiados, la vinculación al ámbito doméstico propio no es la única condición para la discriminación laboral de las mujeres brasileñas, pues de la misma manera las que están exentas de responsabilidades familiares presentan igualmente dificultades en el acceso al mercado de trabajo.

La noción de trabajo conlleva cuestiones como la producción de bienes, transacciones de dinero, circulación de información, desempeño de roles e identidad, etc. Todas ellas son indispensables para el manejo de los diferentes recursos económicos, sociales y personales y pueden medirse tanto en valores materiales como morales ${ }^{8}$. La connotación central básica de lo que es considerado trabajo se argumenta en la idea de que una actividad es directamente aplicada a un objeto ${ }^{9}$ y desarrollarlo implica asumir roles, normas, emplear esfuerzo y técnicas e invertir energía en una interrelación de actividades, relaciones sociales, conocimientos y representaciones que se involucran en los procesos de producción y reproducción. Las ocupaciones laborales tienen un valor material e inmaterial dentro de la sociedad que se asocia a un grado de más o menos prestigio que es asignado a las personas a partir de las actividades que realizan, ya que están inscritas en un marco social y simbólico ${ }^{10}$. Así el trabajo es una expresión política y económica, que en la praxis se manifiesta con múltiples significados y funciones a partir de las

\footnotetext{
6 WALLMAN, Sandra. Introduction, p. 13; ROBERTSON, Alexander F. Beyond the family: the social organization of human reproduction, p. 102; BORDERÍAS, op. cit., p. 58.

7 DALLA COSTA, Mariarosa, JAMES, Selma. El Poder de la Mujer y la Subversión de la Comunidad, p. 34; BORDERÍAS, Cristina, CARRASCO, Cristina. Las mujeres y el trabajo: aproximaciones históricas, sociológicas y económicas, p. 25; COMAS D'ARGEMIR, op. cit., p. 12; MARTíNEZ, Ubaldo. Mujer, trabajo y domicilio, p. 25; NAROTZKY, Susana. Mujer, mujeres, género: una aproximación crítica al estudio de las mujeres en las Ciencias Sociales, p. 150; PARELLA, Sonia. Mujer, inmigrante y trabajadora: la triple discriminación, p. 153.

8 WALLMAN, op. cit., p. 7-8.

9 FIRTH, Raymond. Work and value: reflections on ideas of Karl Marx, p. 178.

${ }^{10}$ COMAS D'ARGEMIR, op. cit., p. 33.
} 
relaciones que se producen entre las diferentes estructuras sociales y de las representaciones e ideologías que en él se involucran ${ }^{11}$.

\section{Aspectos metodológicos}

El trabajo de campo para la investigación se ha desarrollado a partir de la construcción de los relatos de vida de 20 mujeres brasileñas emigradas a Barcelona. Se fundamenta por una parte en entrevistas semiestructuradas realizadas inicialmente a cada una de las mujeres y que posteriormente se han complementado con entrevistas informales, más de 52 en total, a lo largo del periodo de investigación, además de 3 grupos focales. Por otro lado, la observación participante se ha llevado a cabo en contextos cotidianos sociales y privados en los que se involucran las mujeres brasileñas en Barcelona. El contacto con las mujeres colaboradoras se ha focalizado mayoritariamente a través de diversas asociaciones, entidades y agentes de difusión de la cultura brasileña en Barcelona, así como de eventos y celebraciones tanto de iniciativa pública como privada de implicación social y cultural relevante para el trabajo antropológico ${ }^{12}$. La investigación realizada abarca el periodo desde 2013 hasta 2016, lo que ha supuesto la cercanía y en algunos casos la amistad con las mujeres colaboradoras. Los criterios centrales para determinar las mujeres colaboradoras del estudio se han establecido a partir de dos requisitos, ser mujer brasileña viviendo en Barcelona y tener un alto nivel de instrucción, aunque en esta última condición se han tenido en cuenta algunas excepciones.

\section{La emigración como ruptura del proceso de reproducción social}

Como ya he mencionado anteriormente, uno de los aspectos destacados en el estudio es que la emigración aparece como un proyecto autónomo y desvinculado del grupo doméstico en el contexto de origen. Las mujeres que han colaborado en la investigación provienen de grandes ciudades de Brasil y de sus diferentes estados ${ }^{13}$. El 85\%, tienen estudios superiores, de las cuales el $94 \%$ tiene formación universitaria y el $6 \%$ formación profesional, mientras que el $15 \%$, tiene formación básica. Por lo que se refiere al grado de independencia económica antes de emigrar, el 10\% estaba en situación de desempleo y

${ }^{11}$ GODELIER, Maurice. Racionalidad e irracionalidad en economía, p. 263.

${ }^{12}$ Asociación Amigos do Brasil; Asociación Cultural de Capoeira Angola Vadiaçao; Associação de Pesquisadores e Estudantes brasileiros na Catalunha -APEC-; Centro Cultural do Brasil; Consulado Geral do Brasil; Descubra Barcelona - Receptivo Brasileiro; Día de Brasil; Escuela Matumbé Capoeira Barcelona-Roma; Forró Afiado; Forró Baião Brasil; Fuxico Associació Cultural; Mariana Amaral; Nêga Lucas; Roda de Choro de Barcelona; Sarah Pallares; Unidos de Barcelona y Viviendo con Arte, entre otros.

${ }^{13}$ De la Región Nordeste (40\%), se identifican los estados de Bahía (20\%), Ceará (5\%), Maranhão (5\%), Paraíba (5\%) y Pernambuco (5\%). Por lo que se refiere a la Región Sudeste $(55 \%)$, la procedencia de las mujeres es como sigue, Minas Gerais (30\%), São Paolo (15\%) y Rio de Janeiro (10\%). Por último, el 5\% son de Goiás (Región Centro-Oeste). 
vinculada a sus progenitores a nivel familiar y económico, mientras que el 90\% corresponde a trabajadoras independientes económicamente.

Sobre la situación laboral de las mujeres involucradas en el estudio con condición de trabajadoras instruidas, en la etapa pre-migratoria el $83 \%$ trabajaba en sectores de ocupación de acuerdo con su instrucción adquirida, mientras que el 17\% lo hacía en ámbitos laborales distintos a su formación, si bien de forma satisfactoria. Cabe mencionar que a nivel intergeneracional, en los casos de las economías familiares más modestas -a las que pertenecen el $65 \%$ de las colaboradoras-, se aprecia movilidad social ascendente de las mujeres con respecto a sus progenitores. Por otro lado, las mujeres con mayor poder adquisitivo representan el 35\%.

Las razones que llevan a las mujeres a emprender el proyecto de movilidad internacional son diversas y se plantean como la mejora económica, la formación profesional y/o realización de estudios, la evasión de las presiones familiares y/o sociales y la vivencia de nuevas experiencias personales. Si bien en ningún caso existe una única motivación para emigrar, sólo una de ellas aparece como motivación principal y destacada sobre las demás. En esta línea, cabe considerar las condiciones de migración en la idea de articulación de los múltiples aspectos políticos, socioculturales y económicos en las estrategias de las mujeres ${ }^{14}$. Los proyectos que se basan más específicamente en condicionamientos sociales y de autorrealización personal representan el $85 \%$ de los casos, mientras que el 15\% responde a cuestiones principalmente económicas y laborales. Si bien las iniciativas migratorias se definen claramente por su carácter social en la mayoría de los casos, éstas no pueden desvincularse del aspecto económico en la medida en que se trata de un proyecto de subsistencia en la que ambos aspectos se imbrican ${ }^{15}$.

En el momento de la emigración las mujeres se encontraban en situación de independencia personal y económica, así como de estabilidad laboral en el caso de las trabajadoras, lo que facilitó su empoderamiento para emigrar de forma autónoma. Las normas patriarcales, las desigualdades socioeconómicas, y la representación de las imágenes postcoloniales son problemáticas que se manifiestan en los contextos familiar y social como resultado de experiencias opresivas y de subordinación, que influencian la movilidad internacional.

Estas tensiones se producen en primer lugar en relación a los mecanismos de control y subordinación que mediante las relaciones de poder se ejercen en el grupo familiar y en el entorno social respecto a las presiones del sistema patriarcal y su violencia simbólica. Se trata de cuestiones referidas a los roles

\footnotetext{
${ }^{14}$ FELDMAN-BIANCO, Bela, GLICK-SCHILLER, Nina. Una conversación sobre transformaciones de la sociedad, migración transnacional y trayectorias de vida, p. 34.

${ }^{15}$ BORDERÍAS, op. cit., p. 36.
} 
sociales y familiares que se exigen a las mujeres, como casarse y formar una familia a cierta edad y las actitudes, el comportamiento y el trabajo considerado 'femenino', así como otros tipos de relaciones insatisfactorias. Por otra parte, las problemáticas aparecen relacionadas con las desigualdades socioeconómicas. A través de la movilidad se pretenden oportunidades de vida mejores y evitar las experiencias que se consideran insatisfactorias -como el matrimonio y la maternidad en edad temprana-, la precariedad laboral y de vida en general debido a un nivel de instrucción limitado y a los condicionamientos del trabajo familiar, todo ello determinado por la falta de oportunidades que particularmente afectan a las mujeres. En tercer lugar los conflictos hacen referencia a las desigualdades raciales y por ende a la discriminación de clase que se contextualiza en Brasil. La condición de ser afro-brasileña conlleva tener en cuenta el racismo y el sexismo. La presión de los estereotipos sobre las mujeres puede influir en la sobrevaloración de la 'cultura europea' y en la tendencia a europeizar la imagen y el estilo de vida. Por otra parte, en Europa el estereotipo sobre la feminidad de las mujeres brasileñas tiende a ser percibido de manera exótica ${ }^{16}$. En estos casos, la emigración puede percibirse como una mejora de estatus mediante la vivencia europea.

En esta línea, la emigración representa evitar la opresión y dominación familiar y social fundamentada en patrones masculinos y de desigualdad racial ${ }^{17}$. Esta visión social se basa en simbolismos que suscitan deseos de autonomía, de mejora económica y/o de adquisición de nuevas experiencias personales, que influyen en una movilidad fundamentada en las propias iniciativas y en un rol activo y autónomo para transformar las condiciones de existencia a través del proceso migratorio $^{18}$.

\section{La identificación y la sobrecualificación como significante en la devaluación laboral}

En el lugar de acogida, Barcelona, las mujeres brasileñas se insertan en un contexto de simbolismos y percepciones que debe ser considerado. En dichos esquemas sociales y culturales se definen las diferencias y lo que las mujeres simbolizan en dicho entorno respecto a las personas autóctonas. Instituciones e ideologías construyen características distintivas en base a la supuesta identidad atribuida y al lugar de procedencia. Ello conlleva facilitar la inclusión en

\footnotetext{
16 PISCITELLI, Adriana. Looking for New Worlds: Brazilian Women as International Migrants, p. 785.

${ }^{17}$ KLEBA, Teresa. Gênero e Migrações. Trajetórias globais, trajetórias locais de trabalhadoras domésticas, p. 159-160.

${ }^{18}$ HONDAGNEU-SOTELO, Pierrette. Gendered transitions: Mexican experiences of immigration, p. 96; MOROKVASIC, Mirjana. Birds of passage are also women, p. 892; BORDERÍAS, op. cit., p. 28; MARINUCCI, Roberto. Feminization of Migration?, p. 16; ANTÓN, Gemma. La Migración de las Mujeres Brasileñas como Proceso Autónomo: rupturas y (re)Definiciones para la Reproducción Social, p. 53.
} 
ciertos ámbitos y dificultarla en otros a partir de las divisiones sociales y del sistema de producción cristalizadas en el sistema de trabajo, que implica la jerarquización de las personas, de las tareas laborales y de las representaciones sobre todo ello. Es en esta línea que deben contextualizarse las problemáticas en base a los sistemas de género, etnia y clase, así como su articulación con los diferentes aspectos sociales y económicos como categorías asignadas a las mujeres a través de los roles en relación a la división del trabajo. Ello influye en las oportunidades y en las condiciones de acceso a los recursos en base a la segregación en el mercado de trabajo y a las relaciones de poder ${ }^{19}$.

Por lo que se refiere a cómo se inscriben los sentidos dados dentro de dichos simbolismos, significados culturales y relaciones sociales, los aspectos identitarios son así definidos a través de los procesos de trabajo en la medida en que su desarrollo conlleva una experiencia personal y social, a partir de la cual se definen una identidad subjetiva y una identificación social respectivamente. El trabajo es un elemento de valoración de acuerdo a la lógica de mercado que se define en base a los valores sociales dominantes que mantienen las ideologías de la producción ${ }^{20}$. En este sentido, las significaciones corresponden a tipologías sociales, más bien que al aspecto idiosincrático e individual, que tienden a la clasificación de las actividades y a las concepciones sociolaborales, basándose en dimensiones ajenas al trabajo en sí y en la percepción social. En todo ello se involucra la participación de las mujeres brasileñas en las actividades económicas y de trabajo influenciando sus posiciones laborales. Así, considerar las identificaciones previamente a las ocupaciones y al desarrollo del trabajo es contemplar las conceptualizaciones simbólicas definidas y proyectadas socialmente a través del rol laboral ${ }^{21}$.

Los ámbitos en los que se ocupan las mujeres involucradas en Barcelona son principalmente el ámbito del trabajo doméstico, de cuidado y en general del sector servicios. A pesar de que cuentan con un alto nivel de instrucción desarrollan trabajos por debajo de sus habilidades y su formación y, por tanto, desde una posición de subordinación que además tiende a prevalecer en el tiempo. Esta discriminación debe considerarse a partir de mecanismos de exclusión vinculados a ciertas variables estructurales e institucionales del mercado de trabajo que tienen repercusión en las discriminaciones desde la perspectiva de género. En esta línea, cabe mencionar que las políticas migratorias inducen a que las mujeres ocupen los ámbitos de trabajo remunerado feminizados, como respuesta a la demanda existente en el sistema ocupacional español por lo que se refiere a las denominadas 'actividades y

\footnotetext{
${ }^{19}$ COMAS D'ARGEMIR, op. cit., p. 34; PARELLA, op. cit., p. 187.

${ }^{20}$ OFFE, Claus. La Sociedad del trabajo: problemas estructurales y perspectiva de futuro, p. 21; BORDERÍAS, op. cit., p. 124-125.

${ }^{21}$ SCHWIMMER, Erik. The selfand the product: concepts of work in comparative perspective, p. 297.
} 
servicios a la vida diaria', lo que influye en las mujeres migrantes a acceder y permanecer en los ámbitos del trabajo familiar ${ }^{22}$. Por otro lado, tanto el Real Decreto de protección social para empleados/as de hogar ${ }^{23}$ como la Ley de Dependencia ${ }^{24}$ y la Ley Orgánica 3/2007, de 22 de marzo, para la igualdad efectiva de mujeres y hombres ${ }^{25}$, descuidan la intersección de género, clase y etnia al no aplicar medidas efectivas al respecto ${ }^{26}$.

La situación de sobrecualificación en Barcelona, en cuyo concepto contemplo las prácticas del trabajo formal e informal, cobra su significado en la expresión de los patrones de uso de fuerza de trabajo en la estrategia del capital y de las relaciones de poder patriarcales. Ello tiene consecuencias relacionadas con menores salarios, el estatus económico y social, las trayectorias laborales, la movilidad laboral y social, los aspectos psicológicos, la salud, el (des)aprovechamiento del talento y la identidad y sus valores al margen de la instrucción adquirida.

Como he mencionado anteriormente, la situación de sobrecualificación afecta tanto a las mujeres que están vinculadas al trabajo familiar del propio hogar como a las que no tienen dichas responsabilidades, por lo que la sobrecualificación no puede explicarse únicamente en base su articulación con el trabajo asalariado. En ambas situaciones existen casos de mujeres que tienden a ocupar puestos secundarios conllevando su devaluación en el trabajo. En este sentido cabe plantearse que las representaciones sobre ellas persisten en la naturalización de las divisiones sociales del trabajo que son legitimadas y reafirmadas por una parte, en los sistemas patriarcal y capitalista ${ }^{27}$ y por otra, en la etnización de las relaciones sociales y del trabajo basadas en la colonialidad del poder, generando una situación de inferioridad añadida para las mujeres inmigrantes en relación a las mujeres nativas para quienes trabajan ${ }^{28}$.

El trabajo en sobrecualificación se considera cuando el conjunto de las capacidades que posee la persona trabajadora exceden aquellas que son requeridas por el trabajo que desarrolla. Existe cierta ambigüedad a la hora

22 PARELLA, op. cit., p. 211; SOLE, Carlota, CAVALCANTI, Leonardo, PARELLA, Sònia. La inmigración brasileña en la estructura socioeconómica de España, p. 172.

${ }^{23}$ Real Decreto-ley 29/2012 de 28 de diciembre, de mejora de gestión y protección social en el Sistema Especial para Empleados de Hogar y otras medidas de carácter económico y social: $<$ https://www.boe.es/diario_boe/txt.php?id=BOE-A-2012-15764>.

${ }^{24}$ Ley 39/2006, de 14 de diciembre, de Promoción de la Autonomía Personal y Atención a las personas en situación de dependencia: <https://www.boe.es/buscar/act.php?id=BOE-A-2006-21990>.

25 Ley Orgánica 3/2007, de 22 de marzo, para la igualdad efectiva de mujeres y hombres: <https:// www.boe.es/buscar/pdf/2007/BOE-A-2007-6115-consolidado.pdf>.

${ }^{26}$ Código de extranjería 2015: <www.boe.es/legislacion/codigos/>.

${ }^{27}$ HARTMAN, Heidi. Capitalismo, patriarcado y segregación de los empleos por sexo, p. 291.

${ }^{28}$ GUTIÉRREZ-RODRÍGEZ, Encarnación. Trabajo doméstico-trabajo afectivo: sobre heteronormatividad y la colonialidad del trabajo en el contexto de las políticas migratorias de la UE, p. 125-126. 
de definir el concepto de sobrecualificación, ya que en ocasiones suele ser considerada como una forma de 'subocupación' que se determina en relación a las habilidades. Una cuestión que parece consensuada a la hora de abordar el análisis sobre la noción de sobrecualificación es la consideración del aspecto subjetivo. Es decir, debe existir una percepción de trabajar en sobrecualificación por parte de quienes se encuentran en dicha situación, lo que según los/as autores/as mencionados/as se relaciona con sentimientos de insatisfacción y/o el desacuerdo en el desarrollo de los trabajos en cuestión ${ }^{29}$. En el caso de las mujeres brasileñas involucradas, las percepciones subjetivas aparecen como un factor destacable que voy a tratar aquí, si bien tal como voy a argumentar en adelante puede no ser percibido como un aspecto negativo.

Por otra parte, existen algunos estudios que centran su observación en la sobrecualificación y sus consecuencias, si bien no lo hacen aplicando una perspectiva de género. La tendencia en dichos estudios es analizar circunstancias que se dan a partir de situaciones concretas o periodos particulares, como es el caso de las personas que al acabar su formación se incorporan por primera vez al mercado de trabajo, o bien como consecuencia de las crisis económicas ${ }^{30}$. Ello indica que el concepto de sobrecualificación tiende a observarse desde una perspectiva de eventualidad y, por tanto, a considerarse como un fenómeno circunstancial. Contrariamente a estos enfoques, el estudio de las mujeres brasileñas en Barcelona apunta a la sobrecualificación como una situación de prevalencia, por lo que dicho planteamiento lleva a conclusiones diferentes a las de los estudios anteriormente mencionados.

Teniendo en cuenta las consideraciones anteriores, mi perspectiva para el análisis se concretiza en la idea de sobrecualificación a partir de la relación entre el grado de instrucción y las habilidades de las mujeres y, las ocupaciones y tareas que éstas desarrollan por debajo de sus propias capacidades. Ello implica tener como referencia la devaluación laboral que se genera a través del proceso migratorio y su prevalencia, aplicando un punto de vista comparativo entre la situación laboral en Brasil y en Barcelona que evidencia un empeoramiento de las condiciones de trabajo en el contexto de acogida.

El análisis resulta particularmente interesante cuando hablamos de mujeres migrantes en la medida en que existen altas tasas de sobrecualificación en estos casos, ya que mujeres e inmigrantes son grupos mayoritariamente afectados por la sobrecualificación ${ }^{31}$. En España el porcentaje de mujeres inmigrantes en

\footnotetext{
${ }^{29}$ WILKINS, Roger, WOODEN, Mark. Economic approaches to studying underemployment, p. 16-25.

${ }^{30}$ BAERT, Stijn, COCKX, Bart, VERHAEST, Dieter. Overeducation at the start of the career: stepping stone or trap?, p. 131; SÁNCHEZ, María Carmen et alii. Sobrecualificación en tiempos de crisis, p. 590.

${ }^{31}$ SLACK, Tim, JENSEN, Leif. Underemployment among minorities and immigrants, p. 136-137.
} 


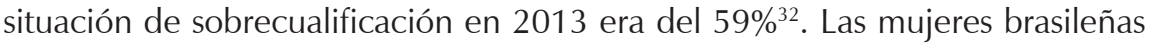
sobrecualificadas que se involucran en el estudio representan el $79 \%$. El 21\% restante de mujeres con alta instrucción trabajan como autónomas.

En base a su nivel de instrucción, de las prácticas laborales que realizan y de su relación entre ambas, la condición de las mujeres colaboradoras en situación de sobrecualificación no encaja en las categorizaciones tradicionales que clasifican a las personas trabajadoras como cualificadas-no cualificadas. Existe un discurso que tiende a definirse a partir de dicha polarización teórica ${ }^{33}$ y de las consideraciones de trabajo cualificado y no cualificado que son definidas a partir del paradigma productivo y de trabajo masculino ${ }^{34}$. Dichos conceptos, cualificado-no cualificado, trascienden para categorizar de la misma manera a las personas trabajadoras. Respecto a las postulaciones sobre la segmentación del mercado de trabajo, la idea de que unas u otras posiciones laborales son ocupadas en relación a tener una formación o a carecer de ella aparece como contradictoria en el caso de las mujeres involucradas en el estudio ${ }^{35}$. La devaluación laboral a través el proceso migratorio no está fundamentada en términos de instrucción y por lo tanto, la desigualdad en el posicionamiento y la remuneración a partir de la división entre personas cualificadas y no cualificadas no es así justificada en estos casos.

Esta perspectiva, contextualizada en los procesos capitalistas, patriarcales y colonizadores, reduce la brecha entre la polaridad cualificación-no cualificación. La sobrecualificación genera una fragmentación respecto a dicha oposición relacionando un nivel de instrucción 'cualificado' con unas prácticas de trabajo que se reconocen como 'no cualificadas'. Ello crea una asimetría respecto a las postulaciones del capital humano en la idea de que tener una mayor instrucción implica obtener mayores ingresos mediante mejores oportunidades de trabajo ${ }^{36}$ ya que la relación entre los aspectos instrucción-ocupación y profesión-prácticas laborales es mediatizada comúnmente por el concepto de cualificación formal, que es reconocido a partir del sistema de clasificación que se define como la adquirida y requerida para el desarrollo de las tareas laborales.

En base a estas argumentaciones y en relación a la cualificación y la participación activa en el mercado de trabajo de las mujeres en cuestión, éstas deberían encontrarse en una posición laboral de acuerdo a los principios que se basan en que el nivel de remuneración es proporcional al del capital

\footnotetext{
${ }^{32}$ Cf. < http://www.oecd.org/els/mig/Indicators-of-Immigrant-Integration-2015.pdf >.

${ }^{33}$ HOREVITZ, Elizabeth. Understanding the anthropology of immigration and migration, p. 749.

34 PARKIN, David. The categorization of work: cases from coastal Kenya, p. 318; BORDERÍAS, op. cit., p. 65.

${ }^{35}$ SINCLAIR, op. cit., p. 9.

${ }^{36}$ BECKER, Gary S. Human capital, p. 59; REQUENA, Félix. La estructura ocupacional de las mujeres en España, p. 36.
} 
humano adquirido, ya que en esta teoría se considera el determinante básico de los ingresos en relación a la ocupación desarrollada ${ }^{37}$. Así, la situación de las mujeres brasileñas sobrecualificadas en Barcelona contradice la teoría del capital humano, ya que dicha teoría no puede explicar la instrucción que encarnan las mujeres en relación a cómo las habilidades son representadas en ellas, ni tampoco los resultados en sus prácticas laborales respecto a su posicionamiento social y sus ingresos económicos ${ }^{38}$.

Esta perspectiva contemporánea sobre la división y la cultura tradicional del trabajo 'femenino' contribuye al entendimiento de los procesos de segregación a partir de los rasgos sexuales y étnicos y al modo en que éstos se feminizan y se jerarquizan. Todo ello conlleva ampliar la perspectiva de análisis en la estructura cualitativa sobre las formas de evaluar las condiciones en las que se desarrolla el trabajo más allá de las dicotomías basadas en las teorías dominantes de mercado y del capital humano. Esto significa visibilizar la discriminación fundamentando que el criterio de referencia no es la falta de cualificación, sino que se trata de la desvalorización en las relaciones y en el mercado de trabajo mediante un proceso por el cual socialmente se utilizan las distinciones de sexo, etnia y clase para la jerarquización de las actividades laborales. Es en este sentido que la sobrecualificación laboral aparece como significante, en la medida en que en ella son condensados los simbolismos sobre cómo las mujeres brasileñas en cuestión son conceptualizadas, posicionadas y los procedimientos para ello.

Así, considerar la problemática de la sobrecualificación laboral abre nuevas vías para el análisis de las categorizaciones sobre el trabajo y las desigualdades posibilitando nuevas perspectivas e interpretaciones. La observación de los casos de las mujeres brasileñas en el contexto de trabajo en Barcelona desde el punto de vista empírico, aporta información cualitativa interesante para el entendimiento sobre la naturaleza de la reproducción de la segregación ocupacional, así como para elucidar el trabajo que realizan y sus condiciones a partir de patrones que amagan las discriminaciones. Las categorizaciones deben traspasar sus límites para evidenciar nuevas formas de 'desigualar' a través de la división del trabajo que afectan especialmente a las mujeres, y que son contextualizadas en la globalización del mercado capitalista, colonialista y patriarcal restando oportunidades para la adquisición de sus derechos ${ }^{39}$.

\footnotetext{
${ }^{37}$ BLACKBURN, Robert M., BROWNE, Jude, BROOKS, Bradley, JARMAN, Jennifer. Explaining Gender Segregation, p. 517.

${ }^{38}$ SINCLAIR, op. cit., p. 12.

${ }^{39}$ POLETTO, Ivo. Migração: direito ou subversão, p. 20-21.
} 


\section{El trabajo vivido y sus significados en la estrategia migratoria}

Existe una dialéctica entre los determinantes de las diversas instituciones y la subjetividad de las mujeres brasileñas, que debe ser entendida en relación al sentido que éstas dan a las prácticas que llevan a cabo en el proceso migratorio. La percepción de las experiencias de trabajo vividas está articulada con el grado de alcance de los objetivos de emigración en relación a sus expectativas. Así, el valor dado al trabajo desarrollado depende en cierta manera de qué experiencias se podrían estar viviendo alternativamente. Ello conlleva centrar la observación teniendo en cuenta unos criterios que se enlazan con contextos que se reconocen fuera del ámbito del trabajo, si bien se involucran en un mismo proceso. Así, el nivel de satisfacción es contrastado en relación al trabajo y a las actividades laborales desarrolladas si bien fuera de sus propios límites, lo que implica interrelacionar las satisfacciones laborales y no laborales.

En esta línea es interesante destacar el significado que el trabajo tiene para las mujeres. Éste puede identificarse como una herramienta principalmente de necesidad económica o de autorrealización siendo ambos aspectos no excluyentes. Ello implica no simplificar el concepto de trabajo desde el punto de vista más puramente económico y basarlo en una relación conceptual trabajo-mercado dentro de la organización de los procesos de producción, pero contemplando los procedimientos de relaciones sociales, habilidades, actitudes, deseos y valores, donde se contextualiza el trabajo en sobrecualificación ${ }^{40}$.

M. tiene 48 años y vive en Terrassa (Barcelona) desde 2005. Seis años después de su llegada se casó con el hombre que hoy es su marido. Desde que llegó a Terrassa M. trabaja haciendo tareas domésticas en varios hogares, siempre los mismos. Cuando su marido se jubiló, redujo su jornada de trabajo a cuatro horas diarias por las mañanas de lunes a viernes para poder pasar más tiempo con él por las tardes. En 2015 su marido tuvo problemas de salud y ello le exige actualmente más dedicación a su cuidado. Por ello, ha reducido a cuatro días semanales su jornada de trabajo fuera de casa. Dice que no tiene una necesidad económica de trabajar, sino más bien personal "yo trabajo para desconectar (...) necesito salir de casa (...) no necesito trabajar para vivir, pero yo necesito trabajar por mí, para no estar parada, para estar con la cabeza ocupada y hacer alguna cosa". Explica que su marido le exige más atención ahora pues aunque es independiente "no quiere quedarse solo". En sus momentos de ocio suele coser, cocinar y también reunirse con sus amigas brasileñas y sus familias, en las que encuentra el apoyo que necesita. La realización de una actividad laboral externa y asalariada cobra su sentido en un acto de individualidad que representa el alejamiento de la rutina de las

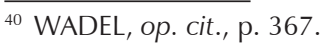


tareas de cuidado y las responsabilidades en el propio hogar ${ }^{41}$. A diferencia de cómo es identificado el trabajo en el hogar en la estructura ocupacional social, para $M$. tiene un significado propio bien diferente que adquiere su sentido en la autorrealización a través del trabajo doméstico asalariado. Ello representa una estrategia para mantener el control de la propia autonomía y la realización personal fracturando la total y única dedicación al trabajo familiar y de cuidado en el propio hogar ${ }^{42}$.

H. tiene 33 años, aunque es licenciada en periodismo, dice: "pero mi trabajo siempre fue cantar". Seis meses después de entrar en la universidad empezó a cantar profesionalmente. Con 19 años, en Brasil, alternaba actividades como periodista, cantante y estudiante. Antes de venir a Barcelona, en 2007, estaba trabajando fuerte con la música en Brasil. Lo que más le costó fue empezar desde cero en Barcelona, ya que no tenía contactos y tuvo que construir su camino de nuevo:

Aquí he trabajado siempre cantando, pero he hecho un montón de cosas, un montón. Porque la música... ahora recién empezaba a sobrevivir de la música pero me ha venido de bajón otra vez, financiero. Es una movida muy inconstante. (...) He hecho masajes en un spa, he trabajado en una empresa de canguros y de limpieza, ya he hecho de azafata, animación, pintar caras de nenes, trabajar en la calle, cara al público...

Hace un año una amiga le habló sobre una oportunidad de trabajo en una escuela de idiomas online, donde actualmente está empleada. H. se encarga de gestionar las incidencias que se generan en relación a los pagos. Está contenta con el trabajo "es un alivio", dice, ya que ello le supone una estabilidad económica que no ha logrado en Barcelona trabajando como cantante. Explica que desarrollar las tareas en su nuevo trabajo le ayuda a mejorar ciertas capacidades como la paciencia y gestionar los conflictos con las personas. Pasa 9 horas sentada y, en ocasiones, puede escuchar música, lo que le genera inspiración para su trabajo musical y le permite potenciar su carrera de cantautora. Explica lo duro que es acostumbrarse al cumplimiento de un horario estricto, algo totalmente nuevo para ella. Para $\mathrm{H}$. su trabajo como cantante se asociada a un modo de vida con el que logra la realización personal ${ }^{43}$. Pero en la imposibilidad de mantener una remuneración continuada y después de realizar durante años diversidad de trabajos precarios, el nuevo empleo representa la oportunidad de un ingreso económico continuado. $\mathrm{Si}$ bien no es el trabajo deseado, es experienciado en términos positivos y de crecimiento, de desarrollo y de mejora de la situación económica así como de ciertas cualidades personales.

\footnotetext{
${ }^{41}$ PINE, Frances. Retreat to the Household? Gendered Domains in Postsocialist Poland, p. 96.

42 BORDERÍAS, op. cit., p. 178.

${ }^{43}$ COMAS D'ARGEMIR, op. cit., p. 12.
} 
Siguiendo en esta línea, cabe considerar que la identidad implica aspectos subjetivos que deben ser entendidos en relación con un contexto más amplio, pues la valoración individual de la posición social se construye en una dialéctica con la estructura donde son creados los tipos de identidad como productos sociales ${ }^{44}$. De este modo, la identidad se define a través del trabajo como experiencia personal dentro de la realidad en la que se contextualizan las mujeres involucradas, donde se identifican y son identificadas ${ }^{45}$.

K., de 36 años, llegó a Barcelona en 2010. Estudió ingeniería metalúrgica en São Carlos, un municipio de São Paulo. Explica qué le empujó a emigrar: "romper vínculos que están forjados en un lugar equivocado, que es un lugar de la dependencia no sólo económica sino dependencia familiar (...) mi búsqueda era tener una identidad". Trabaja en una tienda de ropa infantil 20 horas semanales desde hace más de 5 años, lo que le supone estabilidad económica. Combina dicha ocupación con algunas actuaciones esporádicas como cantante y con el estudio de un postgrado. Su objetivo es mantener un equilibrio entre obtener unos ingresos fijos y poder continuar con sus proyectos con la música. Además, durante los dos últimos años ha trabajado tutorizando cursos trimestrales online sobre materia de género. En la rueda de presentaciones de una asamblea celebrada en Barcelona sobre sociedad, cultura y asociacionismo, en la que nos reunimos más de 25 personas, K. se presenta al resto del grupo como cantante -"sou cantora" - y como tutora de los cursos antes mencionados, si bien, obviando su trabajo como dependienta. Cuando ella explica lo que hace, explica al mismo tiempo lo que es. Así, lo que ella está mostrando ante el resto de personas no es exactamente lo que hace para poder vivir, sino, qué es con lo que ella se siente identificada aunque no sea su principal actividad económica. Las identidades personales están articuladas con las identidades sociales en la idea de reconocerse. A través del tipo de trabajo se construye la identificación social y su estructura de valores, del cual el trabajo es una parte. En la identificación de K., el trabajo que representa el mayor aporte económico para ella resta importancia. Ello evidencia un significado implícito del trabajo en base a unos patrones concretos de identificación ${ }^{46}$.

En estas dinámicas el trabajo se define y es definido a partir del desarrollo de actividades laborales que otorgan satisfacción, si bien en ocasiones pueden no cumplir todas las expectativas, es decir las económicas y las personales. La experiencia de las mujeres con el trabajo se realiza desde una concepción que se contextualiza fuera de los límites de la definición de mercado, si bien por otra parte se fundamenta en ella para construir su propio significado. Es a partir de

\footnotetext{
${ }^{44}$ REQUENA, op. cit., p. 44; TELLEZ, Anastasia. Identidad socioprofesional e identidad de género. Un caso empírico, p. 2.

${ }^{45}$ WALLMAN, op. cit., p. 16; COMAS D'ARGEMIR, op. cit., p. 34.

${ }^{46}$ WALLMAN, op. cit., p. 17.
} 
estas interpretaciones que se adquieren los criterios de valor más allá de la idea de trabajo definida en términos de mercado ${ }^{47}$. Los patrones de identificación con el trabajo cambian dependiendo de las situaciones y las circunstancias. El trabajo y el empleo deben analizarse entonces desde el punto de vista de múltiples estímulos y alicientes, económicos y no económicos, que tienen importancia en los modos de vida y de subsistencia donde las motivaciones se definen entre la obtención de recursos, los valores y las estructuras de identidad ${ }^{48}$.

Los diferentes tipos de trabajo tienen valores distintos en el contexto social y múltiples significados para las mujeres involucradas. Todo ello debe entenderse en relación al proceso migratorio y a las estructuras de poder en términos de relaciones que constituyen las dimensiones de desigualdad en las que se incluye el sistema de trabajo. Los significados dados por las mujeres brasileñas involucradas tienen varias implicaciones con respecto a la noción de trabajo. Por una parte, ponen de manifiesto la concepción de trabajo bajo un denominador común, su función simbólica más allá de la ocupación laboral bajo la lógica económica. Por otra, existe una conceptualización émica que no puede desvinculare del contexto cultural y los significados que en él se otorgan ${ }^{49}$.

\section{Conclusiones}

En la experiencia migratoria de las mujeres brasileñas en Barcelona se relacionan con desigualdades de carácter patriarcal, socioeconómico y racial en su país de origen. Si bien dicho proceso representa obtener nuevas libertades, para las mujeres trabajadoras sobrecualificadas ello supone una devaluación en el contexto del trabajo.

La sobrecualificación laboral en la que se encuentran las mujeres evidencia que su inserción laboral en el contexto de acogida parte de una posición de subordinación. Dicha situación tiende a ser prevalente y a reproducir la segregación en base a nuevos mecanismos que se asocian a las diferencias que se construyen social y simbólicamente y que son representadas en las mujeres, aunque ello no aparece como un conflicto para ellas ni es experienciado como un fracaso, sino que más bien es percibido en base a diferentes niveles de satisfacción.

Las satisfacciones obtenidas a través del trabajo pueden no basarse únicamente en el valor económico, mientras que a su vez se imbrican con otras dimensiones fuera del contexto de trabajo. Autonomía, autoestima, felicidad y ocio son algunos de los aspectos que resultan satisfactorios para

\footnotetext{
${ }^{47}$ Ibidem, p. 5; OFFE, op. cit., p. 23.

${ }^{48}$ WALLMAN, op. cit., p. 6.

${ }^{49}$ COHEN, Anthony P. The Whalsay Croft: traditional work and customary identity in modern times, p. 264-265.
} 
las mujeres y que implican motivaciones para en el desarrollo del trabajo que no se fundamentan únicamente en los ingresos económicos ${ }^{50}$. En esta línea, la sobrecualificación debe analizarse contemplando la perspectiva subjetiva aunque sin relegarlo necesariamente a un aspecto negativo, lo que a su vez implica no dejar de reconocer que es una forma de discriminación que puede ser o no percibida como tal.

Si bien puede considerarse la redefinición de las desigualdades en el contexto de emigración, las diferentes dimensiones en las que se interrelacionan las prácticas de las mujeres permiten entender la construcción de significados en relación a sus estrategias de reproducción social a partir de las acciones que dan sentido a sus trayectorias. La heterogeneidad de significados, percepciones e intereses y la interrelación entre las estructuras, acciones y conflictos que se generan, conlleva a plantearse ir más allá de las limitaciones del concepto de trabajo basado en la perspectiva económica ${ }^{51}$. Es en ese sentido que se requiere de una ética basada en la tolerancia y en la aceptación identitaria para el reconocimiento de la igualdad a todos los niveles y para eliminar las dimensiones genéricas de opresión y desigualdad ${ }^{52}$.

\section{Bibliografía}

ANTÓN, Gemma. La Migración de las Mujeres Brasileñas como Proceso Autónomo: rupturas y (re)Definiciones para la Reproducción Social. Quaderns-e, v. 21, n. 1, 2016, p. 50-62. Disponible en: <http://www.antropologia.cat/quaderns-e-320>. Consultado: 15.12.2016.

BAERT, Stijn; COCKX, Bart; VERHAEST, Dieter. Overeducation at the start of the career: stepping stone or trap? Labor Economics, n. 25, 2013, p. 123-140.

BECKER, Gary S. Human capital. Chicago: The University Press, 1993.

BLACKBURN, Robert M.; BROWNE, Jude; BROOKS, Bradley; JARMAN, Jennifer. Explaining Gender Segregation. British Journal of Sociology, v. 53, n. 4, 2002, p. 513-536.

BORDERÍAS, Cristina. Strategie della libertà: storie e teorie del lavoro femminile. Roma: Manifestolibri, 2000.

BORDERÍAS, Cristina; CARRASCO, Cristina. Las mujeres y el trabajo: aproximaciones históricas, sociológicas y económicas. In BORDERÍAS, Cristina; CARRASCO, Cristina; ALEMANY, Carme (orgs.). Las mujeres y el trabajo. Rupturas conceptuales. Madrid: Icaria, 1994, p. 15-109.

COHEN, Anthony P. The Whalsay Croft: traditional work and customary identity in modern times. In WALLMAN, Sandra (org.). Social anthropology of work. London: Academic Press, 1979, p. 249-267.

\footnotetext{
${ }^{50}$ FIRTH, op. cit., p. 179; OFFE, op. cit., p. 42.

51 OFFE, op. cit., p. 28.

52 LAGARDE, Marcela. Género y feminismo. Desarrollo humano y democracia, p. 205.
} 
COMAS D'ARGEMIR, Dolores. Trabajo, género, cultura: la construcción de desigualdades entre hombres y mujeres. Barcelona: Icaria, 1995.

DALLA COSTA, Mariarosa; JAMES, Selma. El Poder de la Mujer y la Subversión de la Comunidad. México: Siglo XXI, 1977.

FELDMAN-BIANCO, Bela; GLICK-SCHILLER, Nina. Una conversación sobre transformaciones de la sociedad, migración transnacional y trayectorias de vida. Crítica y Emancipación, v. 5, 2011, p. 9-42.

FIRTH, Raymond. Work and value: reflections on ideas of Karl Marx. In WALLMAN, Sandra (org.). Social anthropology of work. London: Academic Press, 1979, p. 177-206.

GODELIER, Maurice. Racionalidad e irracionalidad en economía. 1974.

GUTIÉRREZ-RODRÍGUEZ, Encarnación. Trabajo doméstico-trabajo afectivo: sobre heteronormatividad y la colonialidad del trabajo en el contexto de las políticas migratorias de la UE. Revista de estudios sociales, n. 45, 2013, p. 125126. Disponible en: <http://dx.doi.org/10.7440/res45.2013.10>. Consultado: 13.03.2017.

HARTMAN, Heidi. Capitalismo, patriarcado y segregación de los empleos por sexo. In BORDERÍAS, Cristina; CARRASCO, Cristina; ALEMANY, Carme (orgs.). Las mujeres y el trabajo. Rupturas conceptuales. Madrid: Icaria, 1994, p. 253-294.

HONDAGNEU-SOTELO, Pierrette. Gendered transitions: Mexican experiences of immigration. California: University of California Press, 1994.

HOREVITZ, Elizabeth. Understanding the anthropology of immigration and migration. Journal of Human Behavior in the Social Environment, v. 19, n. 6, 2009, p. 745-758. Disponible en: <http://dx.doi.org/10.1080/10 911350902910914>. Consultado: 30.12.2016.

JULIANO, Dolores. Excluidas y marginales. Madrid: Cátedra, 2004.

KLEBA, Teresa. Gênero e Migrações: trajetórias globais, trajetórias locais de trabalhadoras domésticas. REMHU, Revista Interdisciplinar da Mobilidade Humana, v. 14, n. 26-27, 2006, p. 151-166.

LAGARDE, Marcela. Género y feminismo. Desarrollo humano y democracia. Madrid: horas y HORAS, 1996.

LINO, Nilma. Educação, raça e gênero: relações imersas na alteridade. Cadernos Pagu, n. 6-7, 1996, p. 67-82.

MARINUCCI, Roberto. Feminization of Migration? REMHU, Revista Interdisciplinar da Mobilidade Humana, v. 15, n. 29, 2006, p. 5-22.

MARTÍNEZ, Ubaldo. Mujer, trabajo y domicilio. Barcelona: Icaria, 1995.

MOROKVASIC, Mirjana. Birds of passage are also women. The International Migration Review, v. 18, n. 4, 1984, p. 886-907. Disponible en: <http://www. jstor.org/stable/2546066>. Consultado 02.01.2017.

NAROTZKY, Susana. Antropología económica: nuevas tendencias. Barcelona: Melusina, 2004. 
NAROTZKY, Susana. Mujer, mujeres, género: una aproximación crítica al estudio de las mujeres en las Ciencias Sociales. Madrid: Consejo Superior de Investigaciones Científicas, 1995.

OFFE, Claus. La Sociedad del trabajo: problemas estructurales y perspectiva de futuro. Madrid: Alianza, 1992.

PARELLA, Sonia. Mujer, inmigrante y trabajadora: la triple discriminación. Barcelona: Anthropos, 2003.

PARKIN, David. The categorization of work: cases from coastal Kenya. In WALLMAN, Sandra (org.). Social anthropology of work. London: Academic Press, 1979, p. 317-335.

PINE, Frances. Retreat to the household? Gendered domains in postsocialist Poland. In HANN, Chis M. (org.). Postsocialism: ideals, ideologies and practices in Eurasia. London: Routledge, 2002, p. 95-113.

PISCITELLI, Adriana. Looking for new worlds: Brazilian omen as international migrants. Signs: Journal of Women in Culture and Society, v. 4, n. 33, 2008, p. 784-793.

POLETTO, Ivo. Migração: direito ou subversão. REMHU, Revista Interdisciplinar da Mobilidade Humana, v. 14, n. 26-27, 2006, p. 7-22.

REQUENA, Félix. La estructura ocupacional de las mujeres en España. In RODRIGUEZ, Pilar (org.). Mujeres, trabajos y empleos. Barcelona: Icaria, 2008.

ROBERTSON, Alexander F. Beyond the family: the social organization of human reproduction. Cambridge: Polity Press, 1991.

SÁNCHEZ, María Carmen et alii. Sobrecualificación en tiempos de crisis. Revista venezolana de gerencia, v. 18, n. 64, 2013, p. 584-610.

SINCLAIR, M. Thea. Women, work and skill: economic theories and feminist perspectives. In REDCLIFT, Nanneke; SINCLAIR, M. Thea (orgs.). Working women. International perspectives on labour and gender ideology. New York: Routledge, 1991, p. 1-25.

SLACK, Tim; JENSEN, Leif. Underemployment among minorities and immigrants. In MAYNARD, Douglas C.; FELDMAN, Daniel C. (orgs.). Underemployment: psychological, economic and social challenges. New York: Springer, 2011, p. 127-143.

SOLE, Carlota; CAVALCANTI, Leonardo; PARELLA, Sònia. La inmigración brasileña en la estructura socioeconómica de España. Madrid: Ministerio de trabajo e inmigración, 2011.

SCHWIMMER, Erik. The selfand the product: concepts of work in comparative perspective. In WALLMAN, Sandra (org.). Social anthropology of work. London: Academic Press, 1979, p. 287-315.

TELLEZ, Anastasia. Identidad socioprofesional e identidad de género. Un caso empírico. Gazeta de antropología, n. 18, 2002. Disponible en: < http://hdl. handle.net/10481/7400>. Consultado el 01.03.2017. 
WADEL, Cato. The hidden work of everyday life. In WALLMAN, Sandra (org.). Social Anthropology of Work. London: Academic Press, 1979, p. 365-384.

WALLMAN, Sandra. Introduction. In WALLMAN, Sandra (org.). Social anthropology of work. London: Academic Press, 1979, p. 1-24.

WILKINS, Roger; WOODEN, Mark. Economic approaches to studying underemployment. In MAYNARD, Douglas C.; FELDMAN, Daniel (orgs.). Underemployment: psychological, economic and social challenges. New York: Springer, 2011, p. 13-34.

Recibido para publicación en 31.01.2017

Aceptado para publicación en 02.06.2017 Received for publication in January 315t, 2017 Accepted for publication in June $02^{\text {nd }}, 2017$

ISSN impresso 1980-8585

ISSN eletrônico 2237-9843

http://dx.doi.org/10.1590/1980-85852503880005010 